\title{
hsa_circ_0060975 is highly expressed and predicts a poor prognosis in gastric cancer
}

\author{
PENG XU ${ }^{1,2}$, XIAOLAN XU ${ }^{3}$, LIXIANG ZHANG ${ }^{4}$, ZHENGNAN $\mathrm{LI}^{2}$, \\ JIANJUN QIANG ${ }^{2}$, JIE YAO $^{2}$ and AMAN XU ${ }^{1,4}$ \\ ${ }^{1}$ Department of General Surgery, The Fourth Affiliated Hospital of Anhui Medical University, Hefei, Anhui 230000; \\ Departments of ${ }^{2}$ Hepatobiliary and Pancreatic Surgery and ${ }^{3}$ Critical Care Medicine, Northern Jiangsu People's \\ Hospital Affiliated to Yangzhou University, Yangzhou, Jiangsu 225001; ${ }^{4}$ Department of General Surgery, \\ The First Affiliated Hospital of Anhui Medical University, Hefei, Anhui 230000, P.R. China
}

Received December 16, 2020; Accepted May 24, 2021

DOI: $10.3892 / \mathrm{ol} .2021 .12880$

\begin{abstract}
Gastric cancer (GC) is the fifth most common cancer and GC has a high mortality rate worldwide. Circular (circ) RNAs serve an important role in cancer. The present study aimed to investigate the expression level of hsa_circ_0060975 in gastric cancer (GC) and to determine the clinical pathological significance of hsa_circ_0060975 in patients with GC. Reverse transcription-quantitative PCR was used to detect expression level of hsa_circ_0060975 in 192 GC and adjacent non-cancerous gastric tissues, in GC cell lines (MKN-45, HGC27 and AGS) and a human gastric epithelium cell line (GES-1), as well as in plasma samples from 126 patients with GC and 92 healthy volunteers. All plasma and tissue samples of were obtained from The First Affiliated Hospital of Anhui Medical University (Hefei, China). The relationship between hsa_circ_0060975 expression and clinical pathological factors was analyzed using the $\chi^{2}$ test. The diagnostic value of hsa_circ_0060975 was analyzed using the receiver operating characteristic curve (ROC curve), while the Kaplan-Meier method was used to analyze the relationship of hsa_circ_0060975
\end{abstract}

Correspondence to: Dr Aman Xu, Department of General Surgery, The Fourth Affiliated Hospital of Anhui Medical University, 100 Huaihai Avenue, Hefei, Anhui 230000, P.R. China

E-mail: xuama1965@126.com

Dr Jie Yao, Department of Hepatobiliary and Pancreatic Surgery, Northern Jiangsu People's Hospital Affiliated to Yangzhou University, 98 Nantong West Road, Yangzhou, Jiangsu 225001, P.R. China

E-mail: jieyao1975@126.com

Abbreviations: ROC curve, receiver operating characteristic curve; RT-q, reverse transcription-quantitative; AUC, area under the ROC curve; DFS, disease-free survival; OS, overall survival; CEA, carcinoembryonic antigen; GC, gastric cancer; GO, Gene Ontology; KEGG, Kyoto Encyclopedia of Genes and Genomes

Key words: circular RNAs, hsa_circ_0060975, GC, prognosis expression with the survival of patients with GC as determined by log-rank tests. Univariate and multivariate Cox regression analyses were used to identify the prognostic factors, including hsa_circ_0060975 expression and clinical pathological factors. In addition, the potential function of hsa_circ_0060975 was evaluated via bioinformatics analysis. The expression level of hsa_circ_0060975 was higher in GC tissues compared with adjacent non-cancerous gastric tissues, GC cell lines compared with GES-1 and plasma samples from patients with GC compared with plasma samples from healthy volunteers. In addition, higher hsa_circ_0060975 expression was associated with histological grade, pathological stage and tumor $(\mathrm{T})$ classification in GC tissues and plasma samples $(\mathrm{P}<0.05)$. The area under the ROC curves of hsa_circ_0060975, the combination with hsa_circ_0060975 and carcinoembryonic antigen (CEA) or CEA alone were 0.804 (sensitivity, 0.746; specificity, 0.783; $\mathrm{P}<0.001$ ); 0.931 (sensitivity, 0.937; specificity, 0.870; $\mathrm{P}<0.001$ ) and 0.924 (sensitivity, 0.937; sspecificity, 0.804; $\mathrm{P}<0.001$ ) respectively. The Kaplan-Meier survival analysis revealed that the overall survival (OS) and disease-free survival (DFS) time of patients with higher hsa_circ_0060975 expression were shorter compared with those in patients with lower hsa_circ_0060975 expression. Univariate and multivariate Cox regression analyses in OS and DFS time determined that the expression level of hsa_circ_0060975, histological grade and pathological stage were independent prognostic factors for patients with GC. In addition, the bioinformatics analysis results suggested that the abnormal expression of hsa_circ_0060975 may serve an important role in tumorigenesis. Hence, hsa_circ_0060975 expression may be an independent prognostic factor for patients with GC and may be a potential marker for biological malignancy.

\section{Introduction}

Gastric cancer (GC) is the fifth most common cancer and the third most common cause of cancer mortality worldwide (1). In 2018 , the death rate from gastric cancer was $>8 \%$ of cancer deaths worldwide (2). GC is known to be a highly malignant tumor of the digestive system (1-3). In recent years, the incidence of GC has steadily declined (4), but there are no specific symptoms in early GC, it is often found to metastasized in distant organs, 
such as liver, lymph node metastasis, peritoneal metastasis and lung (1,5-8). Some therapies, such as surgery, radiotherapy and chemotherapy are used to treat GC, but the tumor-free 5-year survival rate of GC is only $20-30 \%$ (9). The combination of gastroscope and biopsy is still considered the gold standard for diagnosing GC, however it is an invasive test (10). The early diagnosis of malignant cancer types can be achieved by using numerous methods, including blood detection, genetic testing and cancer biomarker analysis (11-14), for example, researchers have discovered that long non-coding RNA (lncRNA) in body fluids or multiple serum matrix metalloproteinases with protein biochip technology in gastric cancer can be used as a biomarker for early screening and diagnosis $(13,14)$. In order to improve the early diagnosis of malignant tumors, multiple biomarkers, such as extracellular vesicle and particles (15), urine DNA methylation assay (16), the combination of plasma hsa_circ_0000745 level and CEA (17) have been identified and circular (circ) RNAs have become a research hotspot $(18,19)$.

circRNAs, acting as competitive endogenous RNAs (ceRNAs) and serving an important role in the transcription process, have become key genes for tumor cell proliferation, differentiation, apoptosis and invasion, as well as for cancer diagnosis, survival and metastasis $(20,21)$. For example, circRNA La ribonucleoprotein 4 was found to inhibit cell proliferation and invasion in GC by sponging microRNA (miRNA/miR)-424-5p and regulating large tumor suppressor gene 1 expression (22). In addition, circAKT3 can upregulate phosphoinositide-3-kinase regulatory subunit 1 to enhance cisplatin resistance in GC by sponging miR-198 (23). It has also been demonstrated that hsa_circ_0061140 appears to act as a ceRNA of miR-370, which mediates epithelial-mesenchymal transition by regulating the miR-370/Forkhead Box M1 pathway to promote the proliferation and invasion of ovarian cancer cells (24). In addition, circRNA mannosidase $\alpha$ class $1 \mathrm{~A}$ member 2 was highly expressed in nasopharyngeal carcinoma and was found to be a serum biomarker of malignant tumor (25). A recent study has revealed that upregulated circRNA pleckstrin and sec7 domain containing 3 (circPSD3) has a significant effect on viral RNA abundance in both hepatitis $C$ virus- and dengue virus-infected cells (26). circPSD3 regulates RNA amplification in a viral manner prior to the post-translational step (26), which shows that circRNA may play an unknown important role in the pathogenesis of virus, while eukaryotic translation initiation factor 4A3 shows antiviral properties of the nonsense-mediated decay pathway (26). However, the specific role of circRNAs in tumors is yet to be fully elucidated (27).hsa_circ_0060975 was found to be highly expressed in GC, as detected by the circRNA microarray [data from NCBI Gene Expression Omnibus (GEO) database, GEO accession: GSE122796]. hsa_circ_0060975 is named prostate transmembrane protein androgen Induced 1 and is located at chr20:56223451-56285625 (28). At present, the function of hsa_circ_0060975 in GC remains unknown (28).

The present study aimed to investigate the expression level of hsa_circ_0060975 by reverse transcription-quantitative (RT-q) PCR and to explore the diagnostic and prognostic value of hsa_circ_0060975 in GC. The biological role of hsa_circ_0060975 in gastric cancer was further analyzed by bioinformatics analysis. hsa_circ_0060975 may have certain value in improving the early diagnosis of $\mathrm{GC}$ and provide clues for further exploration of the possible mechanism and targeted therapy of GC.

\section{Materials and methods}

Clinical samples. A total of 192 GC and adjacent non-cancerous gastric tissues were obtained from General Surgery, The First Affiliated Hospital of Anhui Medical University (Hefei, China) between January 2015 and December 2017. The median age of 192 patients with GC was 64 years old (age range, 32-81 years). Among them, 82 patients were male and 110 patients were women. GC tissues and adjacent non-cancerous gastric tissues ( $>5 \mathrm{~cm}$ from the edge of tumor tissue) were obtained from surgical resection specimens. The inclusion criteria of patients with GC were as follows: i) Patients pathologically diagnosed with GC; ii) patients who did not receive radiotherapy and/or adjuvant chemotherapy before surgical resection; iii) patients that reached the D2 surgical resection standard after surgery; and iv) patients with peripheral blood tests performed within 1 week of operation. The exclusion criteria were as follows: i) Patients previously diagnosed with other malignant tumors; ii) patients with tumors previously treated with radiotherapy and/or adjuvant chemotherapy; and iii) patients who died within 4 weeks of the procedure. Written informed consent was obtained from the 192 patients before tissue acquisition, according to the revised Helsinki Declaration. All tissue specimens were immediately frozen in liquid nitrogen after being collected and were stored at $-80^{\circ} \mathrm{C}$ until subsequent use. Between October 2016 and July 2017, blood samples $(4 \mathrm{ml})$ from an additional 126 patients with GC and 92 healthy volunteers were collected from The First Affiliated Hospital of Anhui Medical University (Hefei, China). The median age of an additional 126 patients with plasma samples was 63 years old (age range, $35-78$ years). Of these patients, 56 were male and 70 female. The median age of 92 healthy volunteers with plasma samples was 61 years old (age range, 32-76 years). Of these healthy volunteers, 40 were male and 52 were female. The inclusion and exclusion criteria of an additional 126 patients with GC plasma samples included in the study were the same as those of the 192 patients with GC. The inclusion criteria of healthy volunteers were as follows: i) No abnormality in all indices of physical examination; ii) the same physical examination time as that of an additional 126 patients with GC; and iii) blood samples could be obtained. Exclusion criteria of the healthy volunteers were as follows: i) No history of malignant tumor, chronic inflammation and mental illness; and ii) acute inflammation or recovery period, which may affect the expression of circRNAs (29,30). The blood samples were centrifuged at $1006.2 \mathrm{x}$ g for $15 \mathrm{~min}$ at room temperature and then $4 \mathrm{ml}$ of plasma samples were collected. Plasma samples were frozen in liquid nitrogen immediately after collection and stored at $-80^{\circ} \mathrm{C}$. All patients with GC and healthy volunteers provided written informed consent prior to blood collection.

Clinical information for the patients with GC and healthy volunteers was collected. The Tumor Node Metastasis (TNM) staging of tumor was classified according to the American Joint Committee on Cancer Staging System ( $8^{\text {th }}$ edition) (31). According to WHO's fifth edition of gastric cancer differentiation standard (32), the clinical characteristics and prognosis of patients with GC with well-moderately differentiated and patients with GC with poorly-signet differentiated were different (33-35), 
patients with GC with regards to histological grade were divided into the well-moderately group and poorly-signet group. According to the American Joint Committee on Cancer Staging System ( $8^{\text {th }}$ edition) (31), and the differences in treatment and surgical methods between early gastric cancer group (stage I and II) and advanced gastric cancer group (stage III or higher tumors) (36-40), patients with GC with regards to pathological stage were divided into the early GC group (stage I and II) and advanced GC group (stage III or higher tumors). According to the United States Joint Committee on Cancer Staging System ( $8^{\text {th }}$ edition) (31) and the differences between T3-T4 group and T1-T2 classification group with GC in chemotherapy and surgery (41-43), patients with GC in tumor (T) classification were divided into the T1-T2 and T3-T4 groups.

According to the median of the relative expression level of hsa_circ_0060975 normalized to GAPDH in GC tissues (cut-off value, 8.6) by RT-qPCR, hsa_circ_0060975 expression was divided into a higher expression group and lower expression group in 192 patients with GC. According to the median of the relative expression level of hsa_circ_0060975 normalized to GAPDH in the plasma of patients with GC (cut-off value, 6.1) by RT-qPCR, hsa_circ_0060975 expression was divided into a higher expression group and lower expression group in an additional 126 patients with GC. The present study was approved by the Ethics Committee of Anhui Medical University (approval no. 20150232; Hefei, China).

Follow-up and treatment. Follow-up data were obtained every month by telephone and outpatient services. Follow-up of the present study ended in December 2019. In total, 192 patients with GC were enrolled and these patients reached the D2 surgical resection standard after surgery. In addition, 11 patients in pathologic stage I received no adjuvant chemotherapy, while 181 patients with pathologic stage II or higher tumors received adjuvant chemotherapy. A total of 157 patients received SOX chemotherapy regimen (Oxaliplatin + oral S-1), which was repeated every 3 weeks for 6 cycles and combined chemotherapy was completed within 6 months. Some patients $(n=24)$ could not withstand or rejected intravenous chemotherapy and therefore, received an oral S-1 chemotherapy regimen, which was repeated every 6 weeks for 8 cycles and the oral S-1 chemotherapy regimen was completed in 12 months.

Electro chemiluminescence immunoassay (ECLIA). Concentrations of carcinoembryonic antigen (CEA) from plasma samples were detected by the ECLIA assay kit (cat. no. 157351-03; Roche Diagnostics) using the Roche Cobas E601 Analyzer (Roche Diagnostics). The normal reference value range were $0-5 \mathrm{ng} / \mathrm{ml}$.

Cell culture. GC cell lines (MKN-45, HGC27 and AGS) and a human gastric epithelial cell line (GES-1) were purchased from The Cell Bank of Type Culture Collection of The Chinese Academy of Sciences. GC cell lines (MKN-45, HGC27 and AGS) were cultured in RPMI-1640 medium (Gibco; Thermo Fisher Scientific, Inc.) supplemented with 10\% FBS (HyClone; Cytiva) and 1\% penicillin/streptomycin. GES-1 were cultured in RPMI-1640 medium (Gibco; Thermo Fisher Scientific, Inc.) supplemented with $20 \%$ FBS (HyClone; Cytiva) and $1 \%$ penicillin/streptomycin. All cells were cultured in a humid environment with $5 \% \mathrm{CO}_{2}$ and $95 \%$ air at $37^{\circ} \mathrm{C}$. The cells were cultured in a small dish $(3.5 \mathrm{~cm})$ for $72 \mathrm{~h}$, and then the cells were passaged. After the small dish was full $\left(2 \times 10^{6}\right)$, the total RNA was extracted for RT-qPCR.

$R N A$ extraction and $R T-q P C R$. Total RNA from GC tissues, adjacent non-cancerous gastric tissues, all cell lines and plasma samples were extracted using TRIzol ${ }^{\circledR}$ reagent (Thermo Fisher Scientific, Inc.). According to the manufacturer's instructions, total RNA in plasma samples was extracted using a mirVana PARIS kit (Thermo Fisher Scientific, Inc.). The RNA concentration was determined spectrophotometrically at 260 and $280 \mathrm{~nm}$. Following the manufacturer's protocol, total RNA was reverse transcribed into cDNA using a PrimeScript RT reagent kit with gDNA Eraser (Takara Biotechnology, Co., Ltd.). DyNAmo Flash SYBR Green qPCR kit (Thermo Fisher Scientific, Inc.) was used for qPCR according to the manufacturer's instructions. The thermocycling condition used were as follows: $94^{\circ} \mathrm{C}$ for 3 min for pre-denaturation, followed by 35 cycles of $94^{\circ} \mathrm{C}$ for $30 \mathrm{sec}$ for denaturation, $65^{\circ} \mathrm{C}$ for $30 \mathrm{sec}$ for annealing and $72^{\circ} \mathrm{C}$ for $30 \mathrm{sec}$ for extension. The primers synthesized by Sangon Biotech Co., Ltd. were as follows: hsa_circ_0060975 forward, 5'-TGACATTCTGAAAAGCTGCAA-3' and reverse, 5'-GACT GTCCGCCTTCAGTTCT-3'; and GAPDH forward, 5'-GCACC GTCAAGGCTGAGAAC-3' and reverse, 5'-TGGTGAAGACG CCAGTGGA-3'. GAPDH mRNA expression was used as an internal control. The $2^{-\Delta \Delta C q}$ method was used to calculate mRNA expression (26).

Biological information analysis. hsa_circ_0060975/miRNA target prediction was performed based on Circular RNA interactome database (44). The structure diagram of hsa_circ_0060975 was constructed by circPrimer v.1.2 software (http://www. bioinf.com.cn/) (45). Gene Ontology (GO) analysis and Kyoto Encyclopedia of Genes and Genomes (KEGG) pathway analysis was based on DIANA Tools (TarBase v.8.0 and mirPath v.3.0) (46,47). A network map was drawn using Cytoscape software v.3.7.2 (48). Venn diagram is a diagram for displaying overlapping areas of element sets, in order to clarify the number of overlapping genes in the downstream genes regulated by miRNAs (49). The number of common downstream targets of miRNAs were calculated and drawn using custom Venn diagrams (http://bioinformatics.psb.ugent. be/webtools/Venn/). $\mathrm{P}<0.05$ was used as the criterion for statistical significance.

Statistical analysis. Statistical analysis was performed using SPSS 23.0 (IBM Corp.) software and GraphPad Prism 8.0 (GraphPad Software, Inc.). The data was represented as the mean $\pm \mathrm{SD}$ of 3 independent experiments. The expression levels of hsa_circ_0060975 were compared using a paired or unpaired Student's t-test. Categorical variables were analyzed using a $\chi^{2}$ test. A one-way ANOVA followed by the post hoc Dunnett's test was used to compare the means of $\geq 3$ independent groups. The cut-off value of indexes were performed according to the receiver operating characteristic (ROC) curve using Youden's index (specificity + sensitivity-1). The area under the curve (AUC) size was compared using a z-test. For the survival analysis, the Kaplan-Meier method with a log-rank test and Cox regression method (univariate 


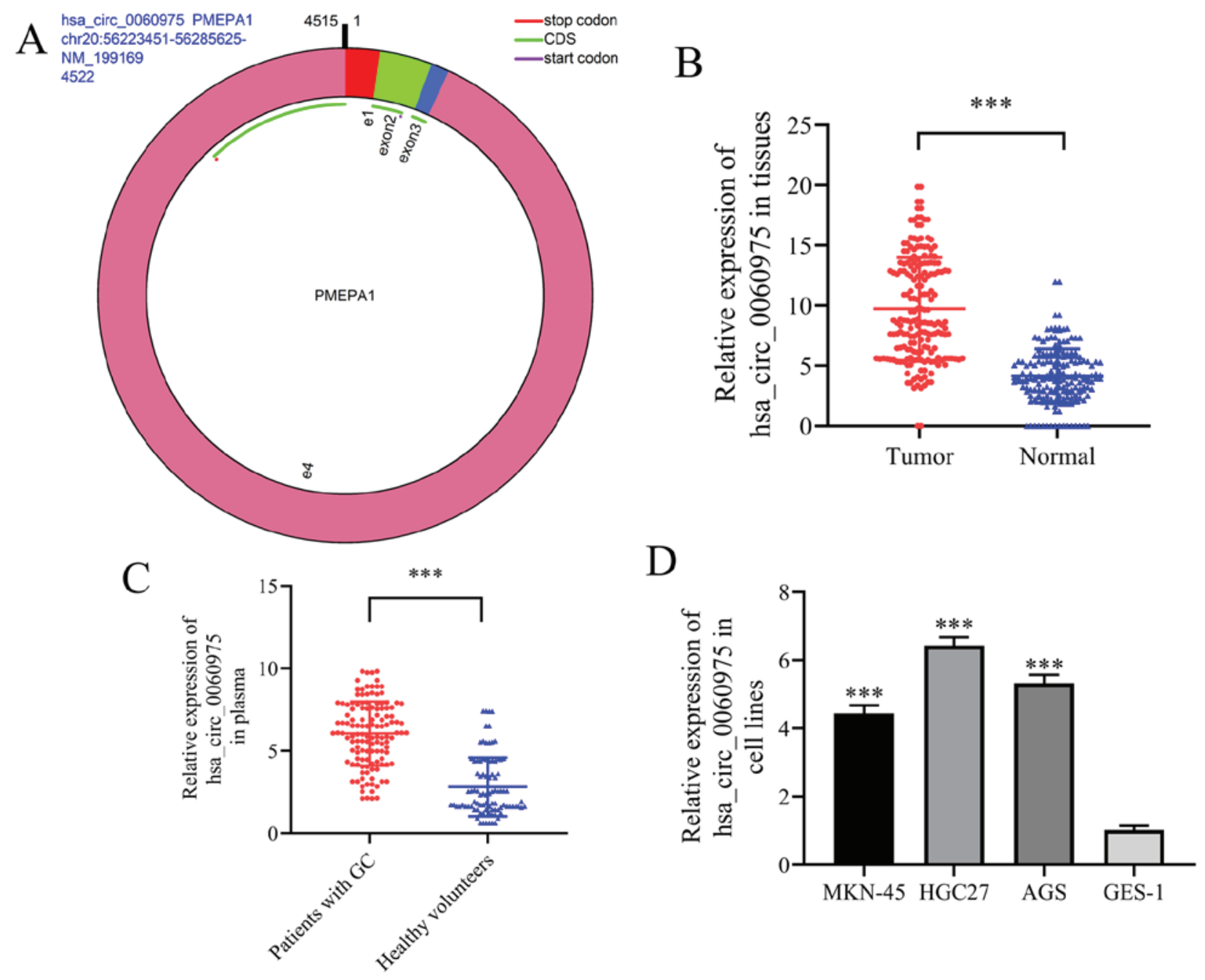

Figure 1.Expression level of hsa_circ_0060975 in GC. (A) Structural diagram of hsa_circ_0060975. (B) Expression level of hsa_circ_0060975 in 192 GC and adjacent normal gastric tissues as detected by RT-qPCR. (C) Expression level of hsa_circ_0060975 in plasma sample of 126 patients with GC and 92 healthy volunteers as detected by RT-qPCR. (D) Expression level of hsa_circ_0060975 in gastric cancer cell lines (MKN-45, HGC27 and AGS) and a human gastric epithelium cell line (GES-1) as detected by RT-qPCR. ${ }^{* * *} \mathrm{P}<0.001$. RT-q, reverse transcription-quantitative; circ, circular RNA; GC, gastric cancer; PMEPA1, Prostate Transmembrane Protein Androgen Induced 1.

and multivariate analyses) were used. $\mathrm{P}<0.05$ was considered to indicate a statistically significant difference.

\section{Results}

Expression level of hsa_circ_0060975 was higher in GC tissues compared with adjacent non-cancerous gastric tissues, GC cell lines compared with GES-1 and plasma samples from patients with GC compared with plasma samples from healthy volunteers. The structure diagram of hsa_circ_0060975 was constructed by circPrimer v.1.2 software (Fig. 1A) (45), and hsa_circ_0060975 expression level was detected in the 192 GC and adjacent non-cancerous normal gastric tissues via RT-qPCR. hsa_circ_0060975 expression was higher in GC tissues (tumor) compared with that in adjacent non-cancerous normal gastric tissues (normal) $(\mathrm{P}<0.001$; Fig. 1B). The expression level of hsa_circ_0060975 in plasma samples was detected in 126 patients with GC and 92 healthy volunteers via RT-qPCR. Compared with the healthy volunteers, a higher hsa_circ_0060975 expression was found in the plasma of patients with GC $(\mathrm{P}<0.001$; Fig. 1C). In addition, the expression level of hsa_circ_0060975 in GC cell lines (MKN-45, HGC27 and AGS) was higher compared with that of a human gastric epithelium cell line (GES-1) $(\mathrm{P}<0.001$; Fig. 1D), these results suggested that hsa_circ_0060975 may promote GC.

Relationshipbetweentheexpressionlevelofhsa_circ_0060975 and clinical pathological parameters. According to the median of the relative expression level of hsa_circ_0060975 normalized to GAPDH in GC tissues, hsa_circ_0060975 expression was divided into a higher expression group and lower expression group. The relationship between the expression level of hsa_circ_0060975 and the clinical pathological features in GC tissue samples is listed in Table I. hsa_circ_0060975 expression with regards to histological grade was significantly different between the well-moderately group and poorly-signet group $(\mathrm{P}<0.001$; Table I), hsa_circ_0060975 expression with regards to pathological stage was significantly different between I + II stage and III stage $(\mathrm{P}<0.001$; Table I), and hsa_circ_0060975 expression in T classification was also significantly different between T1-T2 classification and T3-T4 classification in GC tissues $(\mathrm{P}<0.001$; Table I). 
Table I. Association of hsa_circ_0060975 expression with clinical pathological features in GC tissue samples (n=192) split into the high and low expression groups ( $\mathrm{n}=96$ each).

\begin{tabular}{|c|c|c|c|c|}
\hline \multirow[b]{2}{*}{ Characteristics } & \multirow[b]{2}{*}{$\mathrm{n}$} & \multicolumn{3}{|c|}{ hsa_circ_0060975 } \\
\hline & & $\begin{array}{c}\text { Higher } \\
\mathrm{n}(\%)\end{array}$ & $\begin{array}{c}\text { Lower } \\
\mathrm{n}(\%)\end{array}$ & P-value \\
\hline Age, years & & & & 0.336 \\
\hline$\geq 60$ & 138 & $66(34.37)$ & $72(37.50)$ & \\
\hline$<60$ & 54 & $30(15.63)$ & $24(12.50)$ & \\
\hline Sex & & & & 0.381 \\
\hline Male & 82 & $44(22.92)$ & 38 (19.79) & \\
\hline Female & 110 & $52(27.08)$ & $58(30.21)$ & \\
\hline Alcohol consumption & & & & 0.546 \\
\hline Yes & 68 & $36(18.75)$ & $32(16.67)$ & \\
\hline No & 124 & $60(31.25)$ & $64(33.33)$ & \\
\hline Smoking & & & & 0.540 \\
\hline Yes & 64 & $34(17.71)$ & $30(15.62)$ & \\
\hline No & 128 & $62(32.29)$ & $66(34.38)$ & \\
\hline CEA level, $\mu \mathrm{g} / \mathrm{ml}$ & & & & 0.148 \\
\hline $0-5$ & 90 & $40(20.83)$ & $50(26.04)$ & \\
\hline$>5$ & 102 & $56(29.17)$ & $46(23.96)$ & \\
\hline Histological grade ${ }^{a}$ & & & & $<0.001$ \\
\hline Well-moderately & 76 & $22(11.46)$ & $54(28.13)$ & \\
\hline Poorly-signet & 116 & $74(38.54)$ & $42(21.87)$ & \\
\hline Pathological stage ${ }^{\mathrm{b}}$ & & & & $<0.001$ \\
\hline $\mathrm{I}+\mathrm{II}$ & 78 & $22(11.46)$ & $56(29.17)$ & \\
\hline III & 114 & $74(38.54)$ & $40(20.83)$ & \\
\hline \multicolumn{5}{|l|}{ Lymph node metastasis } \\
\hline No & 66 & $27(14.06)$ & $39(20.31)$ & 0.068 \\
\hline N1-N3 & 126 & $69(35.94)$ & 57 (29.69) & \\
\hline HP infection & & & & 0.773 \\
\hline Positive & 94 & $48(25.00)$ & $46(23.96)$ & \\
\hline Negative & 98 & $48(25.00)$ & $50(26.04)$ & \\
\hline $\mathrm{T}$ classification ${ }^{\mathrm{c}}$ & & & & $<0.001$ \\
\hline $\mathrm{T} 1-\mathrm{T} 2$ & 92 & $22(11.46)$ & $70(36.46)$ & \\
\hline $\mathrm{T} 3-\mathrm{T} 4$ & 100 & $74(38.54)$ & $26(13.54)$ & \\
\hline Tumor size, $\mathrm{cm}$ & & & & 0.062 \\
\hline$<3.5$ & 132 & $72(37.50)$ & $60(31.25)$ & \\
\hline$\geq 3.5$ & 60 & $24(12.50)$ & $36(18.75)$ & \\
\hline
\end{tabular}

${ }^{a}$ According to the differences in treatment and prognosis of patients at different histological grades, patients with GC were

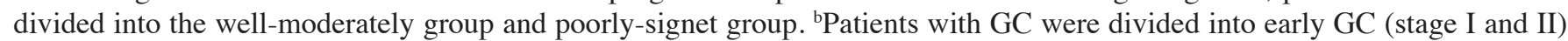
and advanced GC (stage III or higher tumors). ${ }^{\mathrm{c} A c c o r d i n g}$ to the differences in chemotherapy regimens, surgical plans and prognosis of patients with different T classifications, patients with GC were divided into T1-T2 classification and T3-T4 classification. GC, gastric cancer; T, tumor; HP, Helicobacter pylori; CEA, carcinoembryonic antigen.

According to the median of the relative expression level of hsa_circ_0060975 normalized to GAPDH in the plasma of patients with GC, hsa_circ_0060975 expression was divided into a higher expression group and lower expression group. The relationship between the expression level of hsa_circ_0060975 and the clinical pathological parameters in plasma samples is listed in
Table II.hsa_circ_0060975 expression was significantly different among histological grade (well-moderately/poorly-signet; $\mathrm{P}=0.001)$, pathological stage $(\mathrm{I}+\mathrm{II} / \mathrm{III} ; \mathrm{P}=0.001)$ and $\mathrm{T}$ classification (T1-T2/T3-T4; $\mathrm{P}<0.001)$ in the plasma of patients with $\mathrm{GC}$. These results indicated that hsa_circ_0060975 expression may be related to the malignant degree of GC. 
Table II. Association of hsa_circ_0060975 expression with clinical pathological features in GC plasma samples (n=126) split into the high and low expression groups ( $\mathrm{n}=63$ each).

\begin{tabular}{|c|c|c|c|c|}
\hline \multirow[b]{2}{*}{ Characteristics } & \multirow[b]{2}{*}{$\mathrm{n}$} & \multicolumn{3}{|c|}{ hsa_circ_0060975 } \\
\hline & & $\begin{array}{l}\text { Higher } \\
\mathrm{n}(\%)\end{array}$ & $\begin{array}{l}\text { Lower } \\
\mathrm{n}(\%)\end{array}$ & P-value \\
\hline Age, years & & & & 0.413 \\
\hline$\geq 60$ & 94 & $45(35.71)$ & $49(38.89)$ & \\
\hline$<60$ & 32 & $18(14.29)$ & $14(11.11)$ & \\
\hline Sex & & & & 0.720 \\
\hline Male & 56 & $29(23.02)$ & $27(21.43)$ & \\
\hline Female & 70 & $34(26.98)$ & $36(28.57)$ & \\
\hline Alcohol consumption & & & & 0.262 \\
\hline Yes & 44 & $19(15.08)$ & $25(19.84)$ & \\
\hline No & 82 & $44(34.92)$ & $38(30.16)$ & \\
\hline Smoking & & & & 0.262 \\
\hline Yes & 44 & $25(19.84)$ & $19(15.08)$ & \\
\hline No & 82 & $38(30.16)$ & $44(34.92)$ & \\
\hline CEA level, $\mu \mathrm{g} / \mathrm{ml}$ & & & & 0.280 \\
\hline $0-5$ & 72 & $39(30.95)$ & $33(26.19)$ & \\
\hline$>5$ & 54 & $24(19.05)$ & $30(23.81)$ & \\
\hline Histological grade ${ }^{a}$ & & & & 0.001 \\
\hline Well-moderately & 64 & $23(18.25)$ & $41(32.54)$ & \\
\hline Poorly-signet & 62 & $40(31.75)$ & $22(17.46)$ & \\
\hline Pathological stage ${ }^{\mathrm{b}}$ & & & & 0.001 \\
\hline $\mathrm{I}+\mathrm{II}$ & 62 & $22(17.46)$ & $40(31.75)$ & \\
\hline III & 64 & $42(33.33)$ & $22(17.46)$ & \\
\hline Lymph node metastasis & & & & 0.237 \\
\hline N0 & 36 & $15(11.90)$ & $21(16.67)$ & \\
\hline N1-N3 & 90 & $48(38.10)$ & $42(33.33)$ & \\
\hline HP infection & & & & 0.074 \\
\hline Positive & 68 & $29(23.02)$ & $39(30.95)$ & \\
\hline Negative & 58 & $34(26.98)$ & $24(19.05)$ & \\
\hline T classification $^{\mathrm{c}}$ & & & & $<0.001$ \\
\hline $\mathrm{T} 1-\mathrm{T} 2$ & 62 & $19(15.08)$ & $43(34.13)$ & \\
\hline T3-T4 & 64 & $44(34.92)$ & $20(15.87)$ & \\
\hline Tumor size, cm & & & & 0.455 \\
\hline$<3.5$ & 82 & $43(34.13)$ & $39(30.95)$ & \\
\hline$\geq 3.5$ & 44 & $20(15.87)$ & $24(19.05)$ & \\
\hline
\end{tabular}

ROC curve of diagnostic value of hsa_circ_0060975 CEA. CEA was detected using a Roche E601 machine (Roche Diagnostics) with a cut-off value of $5 \mathrm{ng} / \mathrm{ml}$. The analysis of the efficiency of CEA as a diagnostic marker revealed that the area under ROC curve (AUC) was 0.924 (sensitivity, 0.937; sspecificity, 0.804; $\mathrm{P}<0.001$; Fig. $2 \mathrm{~A}$ ). In addition, the analysis of the efficiency of hsa_circ_0060975 as a diagnostic marker demonstrated that the AUC under ROC curve was 0.804 (sensitivity, 0.746; sspecificity, 0.783; P<0.001; Fig. 2A). For the combination of CEA and hsa_circ_0060975, the AUC under ROC curve was 0.931 (sensitivity, 0.937; sspecificity, 0.870; P<0.001; Fig. 2A). The results indicated that CEA and hsa_circ_0060975 may be combined diagnostic markers for GC. 
A

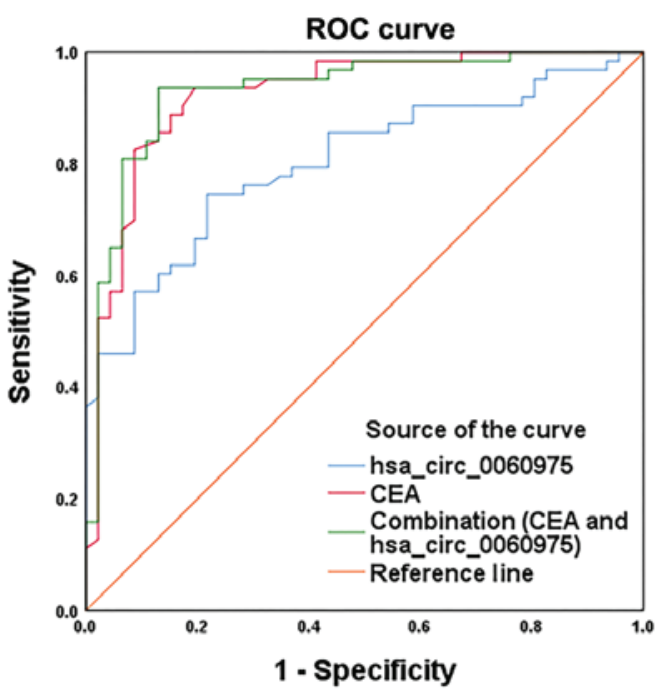

$\mathrm{B}$

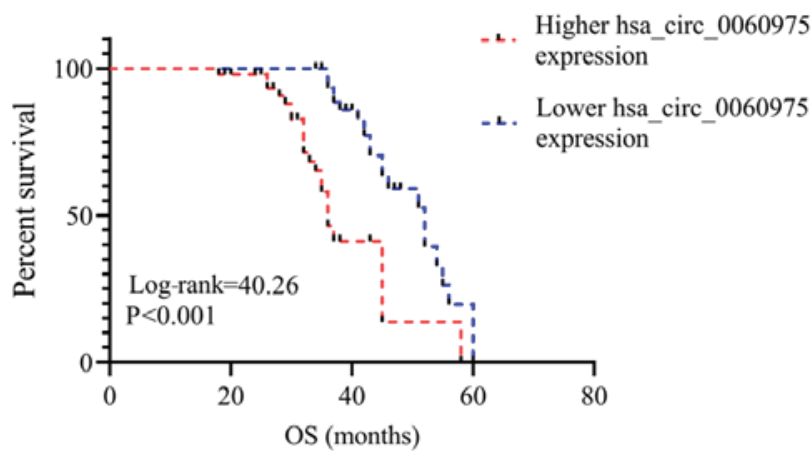

C

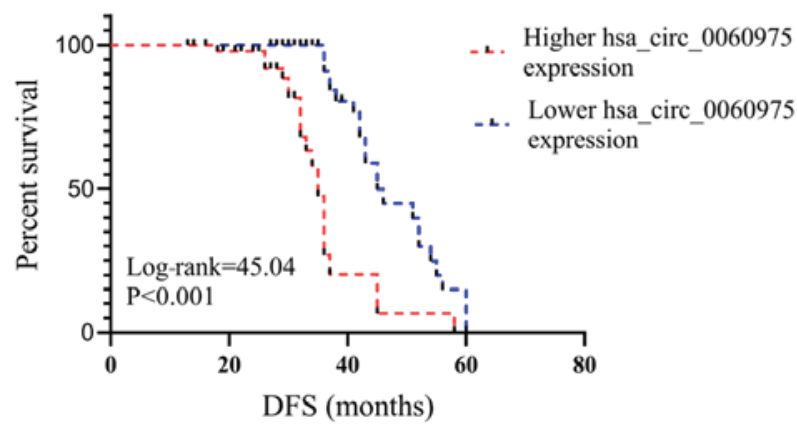

$\mathrm{D}$

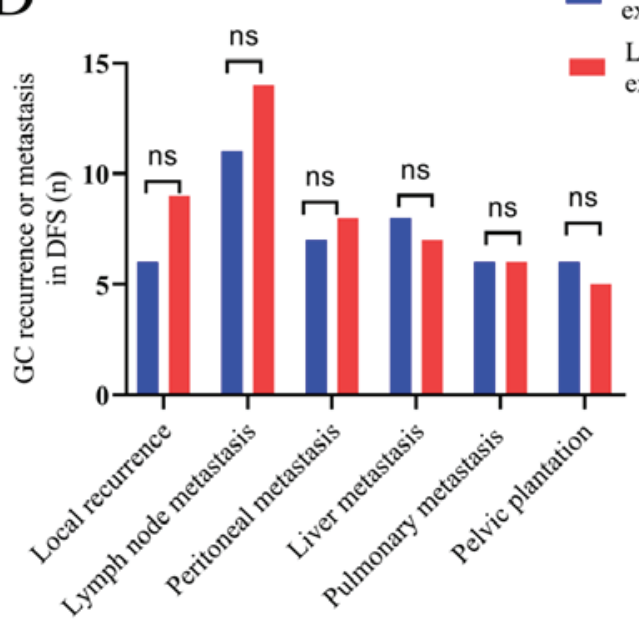

Figure 2. Diagnosis and prognostic value of hsa_circ_0060975 in GC. (A) ROC curves of hsa_circ_0060975, CEA or a combination of hsa_circ_0060975 and CEA. (B) The lower hsa_circ_0060975 expression group showed an improved OS time compared with the higher hsa_circ_0060975 expression groups as determined by using the Kaplan-Meier method and log-rank test. (C) The lower hsa_circ_0060975 expression group showed an improved DFS time compared with the higher hsa_circ_0060975 expression groups, as determined by using the Kaplan-Meier method and log-rank test. (D) Comparative analysis of GC recurrence or metastasis in higher hsa_circ_0060975 expression group $(\mathrm{n}=44)$ and lower hsa_circ_0060975 expression group ( $\mathrm{n}=49)$. circ, circular RNA; ROC, receiver operating characteristic; CEA, carcinoembryonic antigen; OS, overall survival; DFS, disease-free survival; GC, gastric cancer.

Relationshipbetweentheexpressionlevelofhsa_circ_0060975 and prognosis. The follow-up for the present study ended in December 2019. The median survival time was 36.5 months, and 90/192 patients died between 18-60 months. With regards to prognosis, the overall survival (OS) time of patients was analyzed among the 192 patients with GC using the Kaplan-Meier method. The patients with the lower hsa_circ_0060975 expression had an improved prognosis compared with the patients with higher hsa_circ_0060975 expression in OS time (log-rank $=40.26$; $\mathrm{P}<0.001$; Fig. 2B).

Disease-free survival (DFS) time of patients was analyzed among the 192 patients with GC using the Kaplan-Meier method. The patients with the lower hsa_circ_0060975 expression had an improved prognosis compared with the patients with higher hsa_circ_0060975 expression (log-rank $=45.04$; $\mathrm{P}<0.001$; Fig. 2C). Based on DFS time analysis of 192 patients with GC, among the 96 patients in the higher hsa_circ_0060975 expression group, 44 patients had recurrence or metastasis with a median time of 25.5 months, which included 6 patients had with local recurrence (anastomosis or remnant stomach; 6/44), 11 patients with lymph node metastasis (11/44), 7 patients with peritoneal metastasis (7/44), 8 patients with liver metastasis (8/44), 6 patients with pulmonary metastasis (6/44) and 6 patients with pelvic plantation (6/44). In the 96 cases in the lower hsa_circ_0060975 expression group, 49 patients had recurrence or metastasis with a median time of 34.0 months, 9 patients had local recurrence (anastomosis or remnant stomach; 9/49), 14 patients had abdominal lymph node metastasis (14/49), 8 patients had peritoneal metastasis (8/49), 7 patients had liver metastasis (7/49), 6 patients had pulmonary metastasis (6/49) and 5 patients had pelvic plantation (5/49) ( $\mathrm{P}>0.05 ;$ Fig. 2D). The survival time of DFS in the higher hsa_circ_0060975 expression group and lower hsa_circ_0060975 expression group was statistically different, suggesting that patients with GC with lower hsa_circ_0060975 expression had longer DFS time compared with higher hsa_circ_0060975 expression, but there was no statistical difference in the location of recurrence and metastasis.

In addition, based on the clinical data of Table I and the survival status of patients with gastric cancer, univariate and multivariate progression analyses using Cox regression revealed that hsa_circ_0060975 (higher/lower), histological grade (poorly-signet/well-moderately) and pathological 
Table III. Univariate and multivariate Cox regression of proportional hazards model for prediction of overall survival in GC tissue samples.

\begin{tabular}{|c|c|c|c|c|}
\hline Characteristics & $\begin{array}{l}\text { Univariate analysis } \\
\text { HR value }(95 \% \mathrm{CI})\end{array}$ & P-value & $\begin{array}{l}\text { Multivariate analysis } \\
\text { HR value }(95 \% \mathrm{CI})\end{array}$ & P-value \\
\hline Sex (male/female) & $1.036(0.572-1.877)$ & 0.907 & $0.751(0.221-2.552)$ & 0.646 \\
\hline Age, years $(\geq 60 /<60)$ & $0.896(0.464-1.732)$ & 0.984 & $0.974(0.480-2.719)$ & 0.941 \\
\hline Smoking (yes/no) & $1.007(0.535-1.895)$ & 0.984 & $1.283(0.606-2.397)$ & 0.515 \\
\hline Alcohol consumption (yes/no) & $0.971(0.527-1.791)$ & 0.926 & $1.174(0.329-4.189)$ & 0.805 \\
\hline CEA level, $\mu \mathrm{g} / \mathrm{ml}(>5 / 0-5)$ & $1.042(0.575-1.886)$ & 0.892 & $1.015(0.926-1.113)$ & 0.747 \\
\hline \multicolumn{5}{|l|}{ Histological grade } \\
\hline (poorly-signet/well-moderately) & $2.839(1.500-5.374)$ & 0.001 & $3.899(1.753-8.674)$ & 0.001 \\
\hline Pathological stage $^{\mathrm{b}}(\mathrm{III} / \mathrm{I}+\mathrm{II})$ & $3.340(1.657-6.730)$ & 0.001 & $2.744(1.184-6.363)$ & 0.019 \\
\hline HP infection (positive/negative) & $0.707(0.386-1.293)$ & 0.260 & $0.922(0.481-1.767)$ & 0.806 \\
\hline Tumor size, $\mathrm{cm}(\geq 3.5 /<3.5)$ & $0.782(0.403-1.517)$ & 0.467 & $1.111(0.518-2.383)$ & 0.787 \\
\hline hsa_circ_0060975 (higher/lower) & $3.828(2.021-7.250)$ & $<0.001$ & $3.065(1.356-6.925)$ & 0.007 \\
\hline T classificationc (T3-T4/T1-T2) & $3.889(1.852-8.168)$ & $<0.001$ & - & - \\
\hline Lymph node metastasis (yes/no) & $1.188(0.631-2.237)$ & 0.593 & - & - \\
\hline
\end{tabular}

${ }^{a}$ According to the differences in treatment and prognosis of patients of different histological grades, a risk ratio model of poorly-signet vs well-moderately group was constructed. ${ }^{\mathrm{b}}$ A risk ratio model of advanced GC (stage III) vs early GC (stage I and II) group was constructed. ${ }^{\mathrm{c}}$ According to the differences in chemotherapy regimens, surgical plans and prognosis of patients with different $\mathrm{T}$ classifications, a risk ratio model of T3-T4 vs T1-T2 classification group was constructed. HR, hazard ratio; CI, confidence interval; GC, gastric cancer; T, tumor; HP, Helicobacter pylori; CEA, carcinoembryonic antigen.

Table IV. Univariate and multivariate Cox regression of proportional hazards model for prediction of disease-free survival in GC tissue samples.

\begin{tabular}{|c|c|c|c|c|}
\hline Characteristics & $\begin{array}{l}\text { Univariate analysis } \\
\text { HR value }(95 \% \mathrm{CI})\end{array}$ & P-value & $\begin{array}{l}\text { Multivariate analysis } \\
\text { HR value }(95 \% \mathrm{CI})\end{array}$ & P-value \\
\hline Sex (male/female) & $0.951(0.481-1.881)$ & 0.886 & $0.811(0.371-1.772)$ & 0.599 \\
\hline Age, years $(\geq 60 /<60)$ & $1.007(0.535-1.895)$ & 0.984 & $0.811(0.371-1.772)$ & 0.599 \\
\hline Smoking (yes/no) & $0.969(0.504-1.864)$ & 0.925 & $1.024(0.469-2.237)$ & 0.952 \\
\hline Alcohol consumption (yes/no) & $0.909(0.484-1.705)$ & 0.766 & $1.303(0.328-5.180)$ & 0.707 \\
\hline CEA level, $\mu \mathrm{g} / \mathrm{ml}(>5 / 0-5)$ & $0.922(0.503-1.689)$ & 0.793 & $0.629(0.270-1.466)$ & 0.283 \\
\hline \multicolumn{5}{|l|}{ Histological grade ${ }^{\mathrm{a}}$} \\
\hline (poorly-signet/well-moderately) & $3.297(1.749-6.215)$ & 0.001 & $5.480(2.302-13.043)$ & 0.001 \\
\hline Pathological stage $^{\mathrm{b}}(\mathrm{III} / \mathrm{I}+\mathrm{II})$ & $3.368(1.664-6.815)$ & 0.001 & $2.392(1.016-5.633)$ & 0.046 \\
\hline HP infection (positive/negative) & $0.668(0.360-1.239)$ & 0.201 & $1.143(0.512-2.551)$ & 0.744 \\
\hline Tumor size, $\mathrm{cm}(\geq 3.5 /<3.5)$ & $0.852(0.436-1.664)$ & 0.639 & $1.114(0.477-2.601)$ & 0.802 \\
\hline hsa_circ_0060975 (higher/lower) & $4.036(2.125-7.668)$ & $<0.001$ & $4.385(1.761-10.917)$ & 0.001 \\
\hline T classificationc (T3-T4/T1-T2) & $4.804(2.293-10.065)$ & $<0.001$ & - & - \\
\hline Lymph node metastasis (yes/no) & $1.358(0.713-2.585)$ & 0.358 & - & - \\
\hline
\end{tabular}

${ }^{a}$ According to the differences in treatment and prognosis of patients of different histological grades, a risk ratio model of poorly-signet vs well-moderately group was constructed. ${ }^{\mathrm{b}} \mathrm{A}$ risk ratio model of advanced GC (stage III) vs early GC (stage I and II) group was constructed. ${ }^{\mathrm{c}}$ According to the differences in chemotherapy regimens, surgical plans and prognosis of patients with different $\mathrm{T}$ classifications, a risk ratio model of T3-T4 vs T1-T2 classification group was constructed. HR, hazard ratio; CI, confidence interval; GC, gastric cancer; T, tumor; HP, Helicobacter pylori; CEA, carcinoembryonic antigen.

stage (III/I + II) may be significant independent factors in GC $(\mathrm{P}<0.05$; Tables III and IV), indicating the potential of hsa_circ_0060975 as a prognostic biomarker of GC and that high expression of hsa_circ_0060975 may indicate a poor prognosis for patients with GC.
Biological function analysis of hsa_circ_0060975. Further analysis of the potential function of hsa_circ_0060975 and its interaction with miRNA was predicted using the Circular RNA interactome database (44). The result showed that hsa_circ_0060975 interacted with hsa-miR-1299, 
A

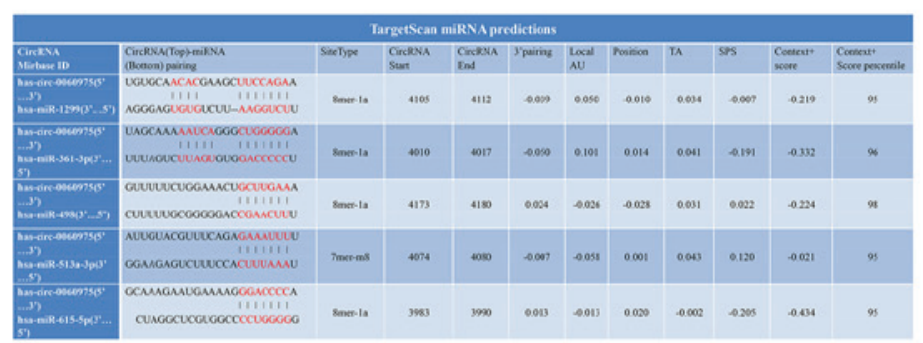

B

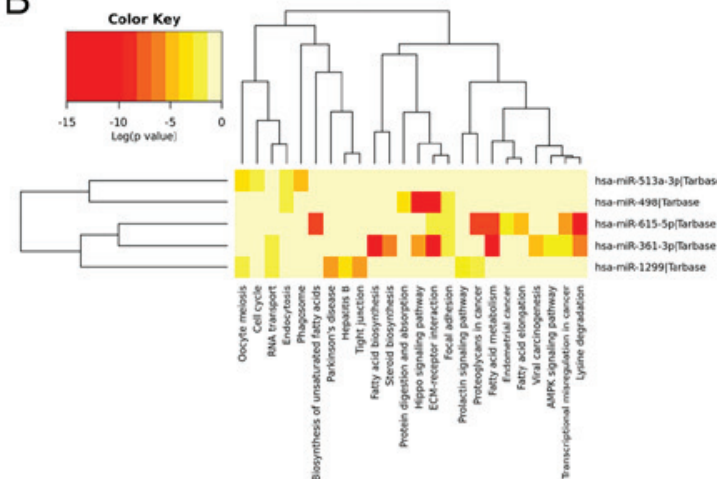

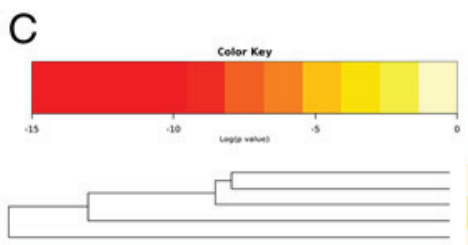
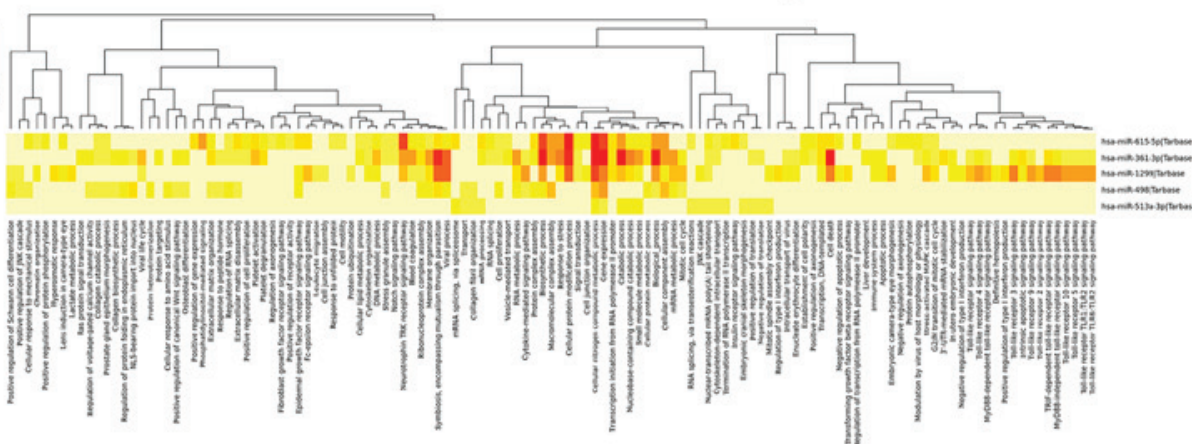

D

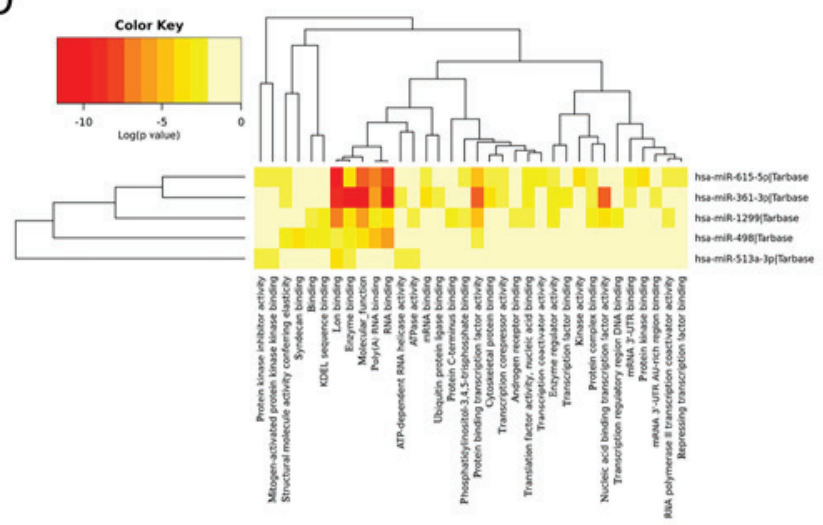

E

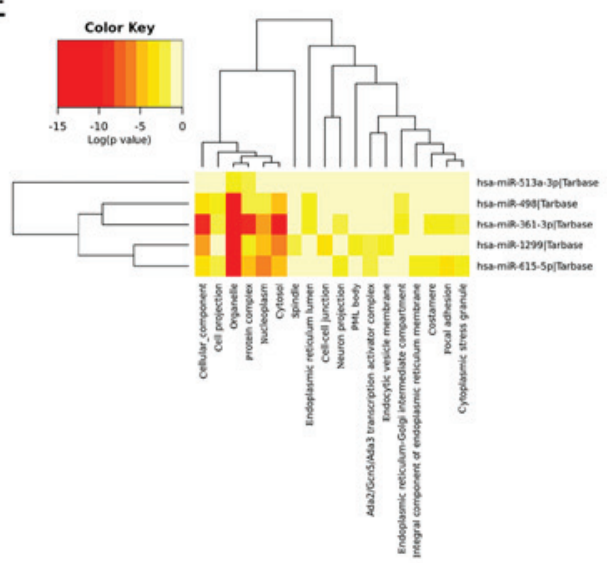

Figure 3. Biological function analysis of hsa_circ_0060975. (A) Predicted hsa_circ_0060975-miRNA interaction (hsa-miR-1299, hsa-miR-361-3p, hsa-miR-498, hsa-miR-513a-3p, and hsa-miR-615-5p) based on Context + score percentile $\geq 90$ by Circular RNA interactome database. (B) KEGG pathway, GO analysis for (C) Biological process, (D) Cellular component and (E) Molecular function analyses results of 5 miRNA related target genes by DIANA Tools (TarBase v8.0 and mirPath v3.0). The color key indicated the $\log _{10} \mathrm{P}$-value, the smaller the $\log _{10} \mathrm{P}$-value the higher the intensity of the red and the smaller the P-value. GO, Gene Ontology; circ, circular RNA; KEGG, Kyoto Encyclopedia of Genes and Genomes; miRNA, microRNA.

hsa-miR-361-3p, hsa-miR-498, hsa-miR-513a-3p and hsa-miR-615-5p based on context + score percentile $\geq 90$ (Fig. 3A). The GO and KEGG pathway analyses results of 5 miRNAs related target genes by DIANA Tools (TarBase v8.0 and mirPath v3 .0) $(46,47)$ (Fig. 3B-E). KEGG pathway analyses results of 5 miRNAs related target genes indicated that the 5 miRNAS were involved in regulating cell cycle, tumor-related signal pathways, involved in cancer transcription regulation, metabolism and other biological functions, such as 'cell cycle', 'AMPK signaling pathway', 'transcriptional misregulation in cancer', 'fatty acid metabolism', 'proteoglycans in cancer' (Fig. 3B). GO analysis (biological process) results of 5 miRNAs related target genes indicated that they were involved in TOLL-like receptor-related signaling pathways, cell proliferation, apoptosis, migration of immune-related inflammatory factors, cell death, DNA and mRNA metabolism, protein modification and other biological functions, such as 'Toll-like receptor receptor 5 signaling pathway', 'cell proliferation', 'negative regulation of apoptotic process', 'leukocyte migration', 'cell death', 'DNA metabolic process', 'RNA metabolic process', 'immune system process' and 'protein ubiquitination' (Fig. 3C). GO analysis (cellular component) results of 5 miRNAs related target genes indicated that they were involved in RNA, protein, and transcription, such as 'protein kinase binding', 'mRNA binding' 'transcription factor binding' (Fig. 3D). GO analysis (molecular function) results of 5 miRNAs related target genes indicated that they were involved in organ-related functions in cells, such as 'cellular component', 'cytosol' 'mucleoplasm', 'organelle', 'endocytic vesicle membrane' etc. (Fig. 3E). 
A

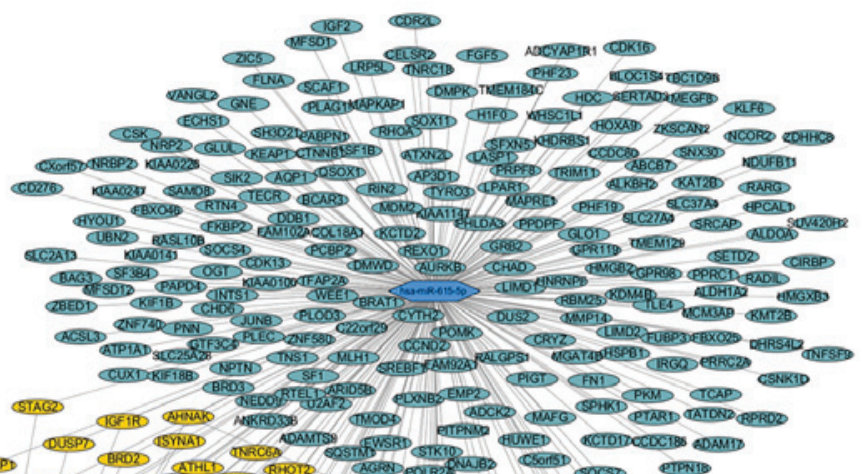

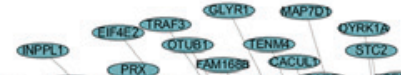

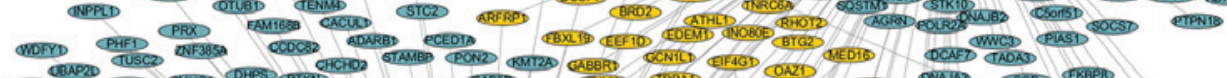

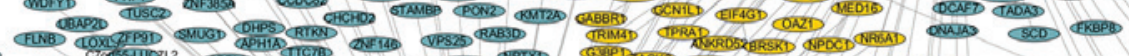

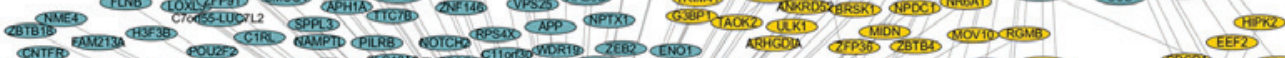

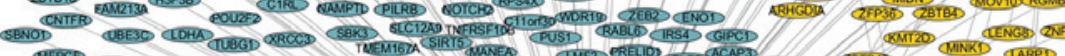

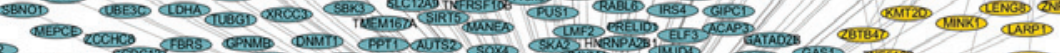

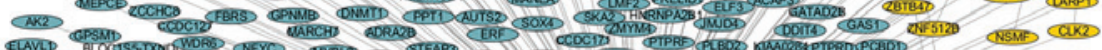

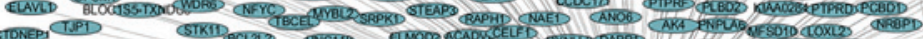

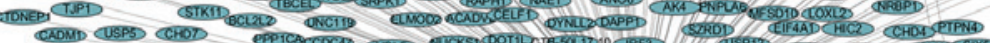

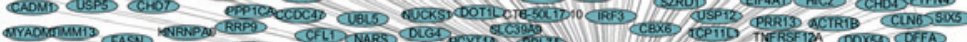

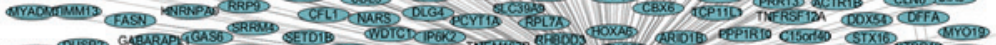

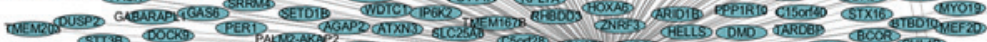

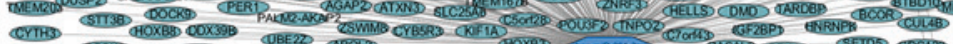

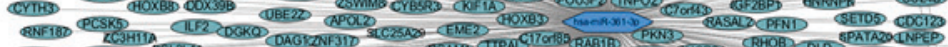

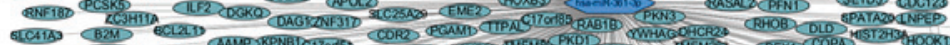

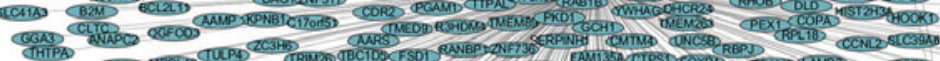

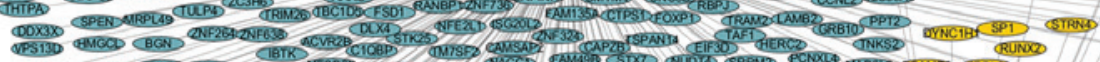

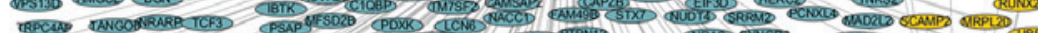

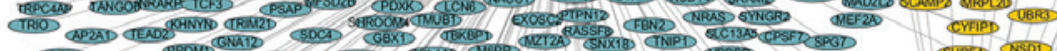

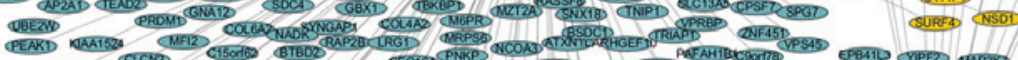

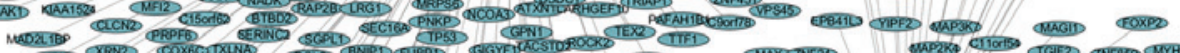

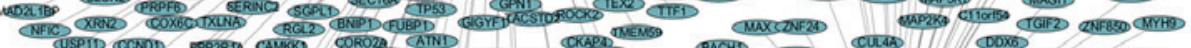

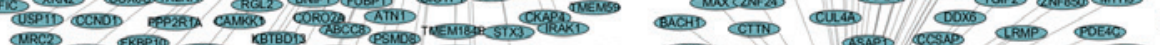

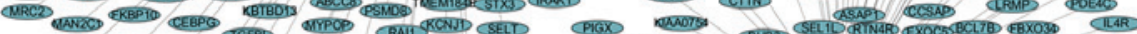

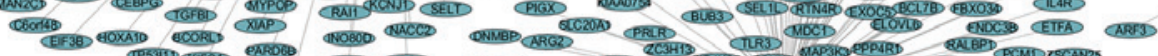

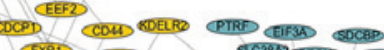

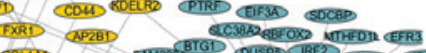

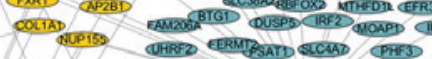

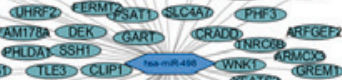

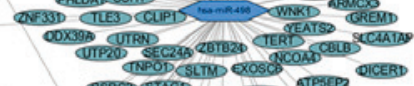

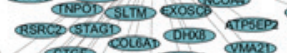

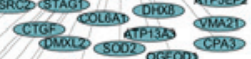

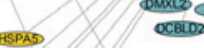

\section{B}

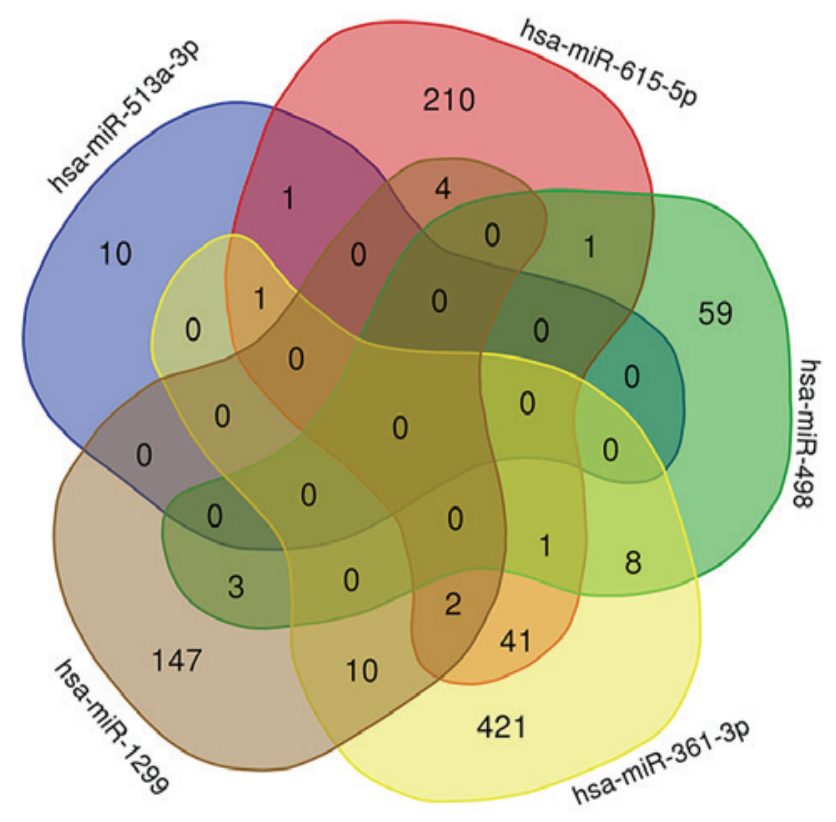

Figure 4. hsa_circ_0060975 related network analysis. (A) A network map comprising hsa_circ_0060975, 5 miRNAs (hsa-miR-1299, hsa-miR-361-3p hsa-miR-498, hsa-miR-513a-3p, and hsa-miR-615-5p) and their downstream target genes generated by Cytoscape v.3.7.2. (B) A Venn diagram revealed the relationship among the number of common downstream targets genes of 5 miRNAs using custom Venn diagrams (http://bioinformatics.psb.ugent.be/webtools/Venn/). miRNA, microRNA; circ, circular RNA. 
In addition, including hsa_circ_0060975, 5 miRNAs (hsa-miR-1299, hsa-miR-361-3p, hsa-miR-498, hsa-miR-513a-3p, and hsa-miR-615-5p) and their downstream target genes generated with Cytoscape v.3.7.2 are presented in Fig. 4A, the results showed that the network relationship of hsa_circ_0060975/5 miRNAs/target genes regulatory axis. Venn diagram is a diagram for displaying overlapping areas of element sets, in order to clarify the number of overlapping genes in the downstream target genes regulated by 5 miRNAs, the Venn diagram demonstrates the number of common downstream targets and overlapping genes in the downstream target genes of the 5 miRNAs (Fig. 4B), suggesting that hsa_circ_0060975 may regulate the biological function of gastric cancer through the circRNA/miRNA/mRNA axis.

\section{Discussion}

It is well known that GC is a highly malignant cancer type (7). At present, several biomarkers for GC have been proposed for diagnosis and prognosis, such as CEA and circulating tumor cells $(50,51)$. Identifying biomarkers for the diagnosis and prognosis of patients with GC are important future research directions (52). As a type of non-coding RNA, circRNAs form a covalently closed continuous ring structure and lack terminal $5^{\prime}$ and $3^{\prime}$ ends and these may be used as new biomarkers for cancer diagnosis and prognosis (23). A previous study found that hsa_circ_0065149 expression was significantly downregulated in GC and lower hsa_circ_0065149 expression was associated with tumor diameter and neural invasion (53). In addition, the lower hsa_circ_0065149 expression group of patients with GC had a longer OS time compared with higher hsa_circ_0065149 expression group (53). It has been previously demonstrated that hsa_circ_0065149 in exosomes was an indicator for early diagnostic and prognosis prediction of GC (53). The expression level of circRNA DNA replication fork stabilization factor DONSON (circDONSON) was found to be associated with tumor node metastasis stage and prognosis and silencing of circDONSON promoted apoptosis, as well as suppressed the proliferation, migration and invasion of BGC-823 and AGS GC cells (54). Higher circRNA protein arginine methyltransferase 5 (circPRMT5) expression was observed in GC and was associated with shorter survival times, while silencing of circPRMT5 inhibited the proliferation and invasion of AGS and MKN-28 GC cells (55). circPRMT5 acts as an oncogene in patients with GC by targeting the miR-145/miR-1304/MYC axis and high circPRMT5 expression may be a poor prognostic indicator for the survival of patients with GC (55). In addition, numerous differentially expressed circRNAs have been reported in tissues (55), blood (56) and saliva (57), indicating circRNAs can be used as biomarkers in multiple diseases, including cancer, such as esophageal cancer and hepatocellular carcinoma (58) and coronary arteries diseases, such as angina pectoris, myocardial infarction and coronary heart disease (59). circRNAs, along with other known biomarkers, may be able to improve the accuracy of the diagnosis of certain diseases, the combination of circRNA panel with CEA and CA19-9 may improve the ability to diagnose colorectal cancer (60) and the combination of plasma hsa_circ_0000745 level and CEA may improve the ability to diagnose GC (17).
The present study demonstrated that the expression level of hsa_circ_0060975 was significantly higher in GC tissues compared with adjacent normal gastric tissues, and was also significantly higher in GC cells compared with GES-1 and plasma samples of patients with GC compared with plasma samples from healthy volunteers. In the present study, hsa_circ_0060975 expression with regards to histological grade was significantly different between the well-moderately group and poorly-signet group in GC tissues and the plasma of patients with GC. In addition, hsa_circ_0060975 expression with regards to pathological stage was significantly different between I + II stage and III stage in GC tissues and the plasma of patients with GC. It was also found that hsa_circ_0060975 expression with regards to $\mathrm{T}$ classification was significantly different between T1-T2 classification and T3-T4 classification in GC tissues and the plasma of patients with GC.

Previous studies have reported that patients with well-moderately differentiated GC and poorly differentiated GC have different sensitivity to chemotherapy regimens and show differences in clinical characteristics, such as poorly differentiated GC has a poor prognosis and a high degree of malignancy $(33,61)$. The treatment effect for poorly differentiated $\mathrm{GC}$ remains poor $(33,61)$ and it has been demonstrated that poorly differentiated GC is a hotspot in clinical treatment research (35). GC is divided into early GC (stage I and II) and advanced GC (stage III or higher tumors) (36-38), and there are differences in treatment and surgical methods between early and advanced GC $(39,40)$. Uzun et al (41) revealed that T1-T2 and T3-T4 classifications of patients with GC showed differences in their clinicopathological characteristics and survival status. T3-T4 classification was an important indicator for patients with GC to choose GC chemotherapy and surgical procedures $(42,43)$. The present study identified that hsa_circ_0060975 was highly expressed in poorly-differentiation levels, advanced gastric cancer (III) and T3-T4 classification. Hence, the expression levels of hsa_circ_0060975 may reflect the degree of malignancy of GC. Based on the aforementioned research results, it was suggested that hsa_circ_0060975 may be a potential marker for evaluating the malignancy of GC and its treatment.

The present study reported the expression level of hsa_circ_0060975 and its diagnostic value in GC. Compared with that diagnostic efficiency of hsa_circ_0060975 or CEA alone, the AUC for the combination of CEA and hsa_circ_0060975 increased to 0.931 and the diagnostic sensitivity and specificity increased to 0.937 and 0.870 , respectively. Hence, this demonstrated an improved diagnostic value,

In the present study, OS and DFS time were shorter in patients with GC with high hsa_circ_0060975 expression compared with patients with GC with low hsa_circ_0060975 expression as detected by the Kaplan-Meier method and log-rank test. Peritoneal transmission is a key factor for poor prognosis and the most common metastatic pattern of GC $(62,63)$. In the present study after follow-up, 7 patients in higher hsa_circ_0060975 expression group with GC had peritoneal metastasis (7/44), while 8 patients in lower hsa_circ_0060975 expression group had peritoneal metastasis (8/49), and there was no significant difference in peritoneal metastasis between the high hsa_circ_0060975 expression and low hsa_circ_0060975 expression groups. There are numerous factors that affect the 
prognosis of patients with GC $(64,65)$. Recent studies have found that positive abdominal cytology is an important factor affecting the prognosis of patients with GC $(66,67)$. A recent study found postoperative chemotherapy can improve the survival rate of GC patients with positive peritoneal cytology (68).

In the present study, univariate and multivariate Cox analyses demonstrated that hsa_circ_0060975 (higher/lower), histological grade (poorly-signet/well-moderately) and pathological stage (III/I + II) were independent prognostic factors in patients with GC. Hence, the finding of the present study indicated that hsa_circ_0060975 (higher), histological grade (Poorly-signet) and pathological stage (III) act as predictors of poor prognosis in patients with GC.

Recent studies have shown that circRNAs can regulate the function of miRNA via sponging $(69,70)$. miRNA has a positive or negative regulatory effect on downstream target genes $(71,72)$. A number of studies have revealed that the circRNA/miRNA/mRNA axis is involved in the malignant process of tumors $(72,73)$. The present study used bioinformatics methods to analyze hsa_circ_0060975 and predict the regulatory effects of miRNA and downstream target genes. GO and KEGG analyses of downstream target gene using DIANA Tools (TarBase v8.0 and mirPath v3.0) $(46,47)$ revealed numerous cancer-related biological regulations, such as 'cell cycle', 'transcriptional misregulation in cancer' and 'cell proliferation' amongst others. In addition, it was found these participate in other biological functions, such as 'immunity' and 'metabolism'. The bioinformatics analysis performed in the present study demonstrated that the abnormal expression of hsa_circ_0060975 may serve an important role in the occurrence of GC and hsa_circ_0060975 may regulate the biological function of gastric cancer through the circRNA/miRNA/mRNA axis.

However, due to the limited number of samples, the value and mechanism of hsa_circ_0060975 in GC needs to be further studied. In addition, the present was a retrospective study, which only used GC tissues and blood samples and did not obtain detailed data about peritoneal cytology, which may have an impact on the prognosis of patients with GC $(66,67)$. Future research should collect detailed patient data, including abdominal cytology data of patients with GC and conduct large-scale and multicenter studies to investigate the value and possible mechanism of hsa_circ_0060975 in patients with GC.

In conclusion, the present study demonstrated that hsa_circ_0060975 expression was an independent prognostic factor for patients with GC and may be a potential marker for biological malignancy. The present study demonstrated that the combination of CEA and hsa_circ_0060975 may improve the diagnosis patients with GC and provide clues for further exploration of the possible underlying mechanism and targeted therapy for GC.

\section{Acknowledgements}

Not applicable.

\section{Funding}

This work was supported by Fund of National Natural Science Foundation of China (grant no. 81772516) and the National and Anhui Provincial Key Clinical Specialty Discipline Construction Program (grant no. Z155080000004).

\section{Availability of data and materials}

The datasets used and/or analyzed during the current study are available from the corresponding author on reasonable request.

\section{Authors' contributions}

PX, XX, JY and AX performed the experiments and contributed equally to the study design, as well as drafting and revision of the manuscript. $\mathrm{LZ}, \mathrm{ZL}$ and JQ performed the analysis. AX and JY confirmed the authenticity of all the raw data. All authors read and approved of the final manuscript.

\section{Ethics approval and consent to participate}

The study was approved by Ethics Committee of Anhui Medical University (approval no. 20150232; Hefei, China). Each patient provided signed informed consent.

\section{Patient consent for publication}

Not applicable.

\section{Competing interests}

The authors declare that they have no competing interests.

\section{References}

1. Erratum: Global cancer statistics 2018: GLOBOCAN estimates of incidence and mortality worldwide for 36 cancers in 185 countries. CA Cancer J Clin 70: 313, 2020

2. Hamashima C: The burden of gastric cancer. Ann Transl Med 8: $734,2020$.

3. Yang L, Ying X, Liu S, Lyu G, Xu Z, Zhang X, Li H, Li Q, Wang $\mathrm{N}$ and Ji J: Gastric cancer: Epidemiology, risk factors and prevention strategies. Chin J Cancer Res 32: 695-704, 2020.

4. Eusebi LH, Telese A, Marasco G, Bazzoli F and Zagari RM: Gastric cancer prevention strategies: A global perspective. J Gastroenterol Hepatol 35: 1495-1502, 2020.

5. Dong D, Fang MJ, Tang L, Shan XH, Gao JB, Giganti F, Wang RP, Chen X, Wang XX, Palumbo D, et al: Deep learning radiomic nomogram can predict the number of lymph node metastasis in locally advanced gastric cancer: An international multicenter study. Ann Oncol 31: 912-920, 2020.

6. Verstegen MH, Harker M, van de Water C, van Dieren J, Hugen N, Nagtegaal ID, Rosman C and van der Post RS: Metastatic pattern in esophageal and gastric cancer: Influenced by site and histology. World J Gastroenterol 26: 6037-6046, 2020.

7. Chen D, Liu Z, Liu W, Fu M, Jiang W, Xu S, Wang G, Chen F, Lu J, Chen $\mathrm{H}$, et al: Predicting postoperative peritoneal metastasis in gastric cancer with serosal invasion using a collagen nomogram. Nat Commun 12: 179, 2021.

8. Siegel RL, Miller KD and Jemal A: Cancer statistics, 2019. CA Cancer J Clin 69: 7-34, 2019.

9. Zhao TT, Xu H, Xu HM, Wang ZN, Xu YY, Song YX, Yin SC Liu XY and Miao ZF: The efficacy and safety of targeted therapy with or without chemotherapy in advanced gastric cancer treatment: A network meta-analysis of well-designed randomized controlled trials. Gastric Cancer 21: 361-371, 2018.

10. Virgilio E, Proietti A, D'Urso R, Cardelli P, Giarnieri E, Montagnini M, Giovagnoli MR, Mercantini P, Balducci G and Cavallini M: Measuring Intragastric Tumor Markers in Gastric Cancer Patients: A Systematic Literature Review on Significance and Reliability. Anticancer Res 37: 2817-2821, 2017. 
11. Bo H, Fan L, Gong Z, Liu Z, Shi L, Guo C, Li X, Liao Q, Zhang $\mathrm{W}$, Zhou M, et al: Upregulation and hypomethylation of lncRNA AFAP1 AS1 predicts a poor prognosis and promotes the migration and invasion of cervical cancer. Oncol Rep 41: 2431-2439, 2019.

12. Bo H, Fan L, Li J, Liu Z, Zhang S, Shi L, Guo C, Li X, Liao Q, Zhang W, et al: High Expression of lncRNA AFAP1-AS1 Promotes the Progression of Colon Cancer and Predicts Poor Prognosis. J Cancer 9: 4677-4683, 2018.

13. Yuan L, Xu ZY, Ruan SM, Mo S, Qin JJ and Cheng XD: Long non-coding RNAs towards precision medicine in gastric cancer: Early diagnosis, treatment, and drug resistance. Mol Cancer 19: 96, 2020.

14. Liu J, Zhou L, Lin S and Yao B: Role of serum matrix metalloproteinase in the diagnosis of gastric cancer. Pak J Med Sci 36: $1025-1031,2020$

15. Hoshino A, Kim HS, Bojmar L, Gyan KE, Cioffi M, Hernandez J, Zambirinis CP, Rodrigues $\mathrm{G}$, Molina $\mathrm{H}$, Heissel S, et al: Extracellular Vesicle and Particle Biomarkers Define Multiple Human Cancers. Cell 182: 1044-1061.e18, 2020.

16. Chen X, Zhang J, Ruan W, Huang M, Wang C, Wang H, Jiang Z Wang S, Liu Z, Liu C, et al: Urine DNA methylation assay enables early detection and recurrence monitoring for bladder cancer. J Clin Invest 130: 6278-6289, 2020

17. Huang M, He YR, Liang LC, Huang Q and Zhu ZQ: Circular RNA hsa_circ_0000745 may serve as a diagnostic marker for gastric cancer. World J Gastroenterol 23: 6330-6338, 2017.

18. Wang Y, Li Z, Xu S and Guo J: Novel potential tumor biomarkers: Circular RNAs and exosomal circular RNAs in gastrointestinal malignancies. J Clin Lab Anal 34: e23359, 2020.

19. Xie Y, Li J, Li P, Li N, Zhang Y, Binang H, Zhao Y, Duan W, Chen Y, Wang Y, et al: RNA-Seq Profiling of Serum Exosomal Circular RNAs Reveals Circ-PNN as a Potential Biomarker for Human Colorectal Cancer. Front Oncol 10: 982, 2020.

20. Bachmayr-Heyda A, Reiner AT, Auer K, Sukhbaatar N, Aust S, Bachleitner-Hofmann T, Mesteri I, Grunt TW, Zeillinger R and Pils D: Correlation of circular RNA abundance with proliferation - exemplified with colorectal and ovarian cancer, idiopathic lung fibrosis, and normal human tissues. Sci Rep 5: 8057, 2015.

21. Chen G, Shi Y, Zhang Y and Sun J: circRNA_100782 regulates pancreatic carcinoma proliferation through the IL6-STAT3 pathway. OncoTargets Ther 10: 5783-5794, 2017

22. Zhang J, Liu H, Hou L, Wang G, Zhang R, Huang Y, Chen X and Zhu J: Circular RNA_LARP4 inhibits cell proliferation and invasion of gastric cancer by sponging miR-424-5p and regulating LATS1 expression. Mol Cancer 16: 151, 2017.

23. Huang X, Li Z, Zhang Q, Wang W, Li B, Wang L, Xu Z, Zeng A, Zhang X, Zhang X, et al: Circular RNA AKT3 upregulates PIK3R1 to enhance cisplatin resistance in gastric cancer via miR-198 suppression. Mol Cancer 18: 71, 2019.

24. Chen Q, Zhang J, He Y and Wang Y: hsa_circ_0061140 knockdown reverses FOXM1-mediated cell growth and metastasis in ovarian cancer through miR-370 sponge activity. Mol Ther Nucleic Acids 13: 55-63, 2018.

25. Fan CM, Wang JP, Tang YY, Zhao J, He SY, Xiong F, Guo C, Xiang B, Zhou M, Li XL, et al: circMAN1A2 could serve as a novel serum biomarker for malignant tumors. Cancer Sci 110 : 2180-2188, 2019.

26. Chen TC, Tallo-Parra M, Cao QM, Kadener S, Böttcher R, Pérez-Vilaró G, Boonchuen P, Somboonwiwat K, Díez J and Sarnow P: Host-derived circular RNAs display proviral activities in Hepatitis C virus-infected cells. PLoS Pathog 16: e1008346, 2020.

27. Goodall GJ and Wickramasinghe VO: RNA in cancer. Nat Rev Cancer 21: 22-36, 2021.

28. Glažar P, Papavasileiou P and Rajewsky N: circBase: A database for circular RNAs. RNA 20: 1666-1670, 2014.

29. Liu G, Wan Q, Li J, Hu X, Gu X and Xu S: circ 0038467 regulates lipopolysaccharide-induced inflammatory injury in human bronchial epithelial cells through sponging miR-338-3p. Thorac Cancer 11: 1297-1308, 2020.

30. Zhang L, Han B, Liu H, Wang J, Feng X, Sun W, Cai D, Jia H and Jiang D: Circular RNA circACSL1 aggravated myocardial inflammation and myocardial injury by sponging miR-8055 and regulating MAPK14 expression. Cell Death Dis 12: 487, 2021.

31. Amin MB, Edge S, Greene F, Byrd DR, Brookland RK, Washington MK et al (eds.): Gastrointenstinal Stromal Tumor In: The 8th edition of the AJCC Cancer Staging Manual. Springer, New York, NY, pp523 529, 2017.

32. Nagtegaal ID, Odze RD, Klimstra D, Paradis V, Rugge M, Schirmacher $\mathrm{P}$, Washington KM, Carneiro F and Cree IA; WHO Classification of Tumours Editorial Board: The 2019 WHO classification of tumours of the digestive system. Histopathology 76: 182-188, 2020.
33. Sun LB, Zhao GJ, Ding DY, Song B, Hou RZ and Li YC: Comparison between better and poorly differentiated locally advanced gastric cancer in preoperative chemotherapy: A retrospective, comparative study at a single tertiary care institute. World J Surg Oncol 12: 280, 2014

34. Hirai H, Yoshizawa T, Morohashi S, Haga T, Wu Y, Ota R, Takatsuna M, Akasaka H, Hakamada $\mathrm{K}$ and Kijima $\mathrm{H}$ Clinicopathological significance of gastric poorly differentiated medullary carcinoma. Biomed Res 37: 77-84, 2016.

35. Chen JN, Wang QW, Zhang QW, Tang ZR and Li XB: Poorly differentiated is more significant than signet ring cell component for lymph node metastasis in mixed-type early gastric cancer: A retrospective study from a large-volume hospital. Surg Endosc 35: 1558-1565, 2021.

36. Zhang M, Fan Y, Che X, Hou K, Zhang C, Li C, Wen T, Wang S, Cheng Y, Liu Y, et al: 5-FU-Induced Upregulation of Exosomal PD-L1 Causes Immunosuppression in Advanced Gastric Cancer Patients. Front Oncol 10: 492, 2020.

37. Schultz KS, de Geus SWL, Sachs TE, Morgan RB, Ng SC, McAneny D and Tseng JF: Influence of race and sociodemographic factors on declining resection for gastric cancer: A national study. Am J Surg 221: 155-161, 2021

38. Chen WM, Liu JL, Chuang HC, Chang YL, Yeh CM, Wu CS and Wu SF: Helios Expression in Tumor-Infiltrating Lymphocytes Correlates with Overall Survival of Advanced Gastric Cancer Patients. Life (Basel) 10: 189, 2020.

39. Wang X, Liu Y, Niu Z, Fu R, Jia Y, Zhang L, Shao D, Du H, $\mathrm{Hu}$ Y, Xing X, et al: Prognostic value of a 25-gene assay in patients with gastric cancer after curative resection. Sci Rep 7: $7515,2017$.

40. Marcos P, Brito-Gonçalves G, Libânio D, Pita I, Castro R, Sá I, Dinis-Ribeiro M and Pimentel-Nunes P: Endoscopic grading of gastric intestinal metaplasia on risk assessment for early gastric neoplasia: Can we replace histology assessment also in the West? Gut 69: 1762-1768, 2020 .

41. Uzun O, Gulmez S, Senger AS, Percem A, Polat E and Duman M: Prognostic Factors in Operated T3-T4 Gastric Cancer. J Coll Physicians Surg Pak 30: 1047-1052, 2020.

42. Petrioli R, Marrelli D, Roviello F, D'Ignazio A, Torre P, Chirra M, Savelli V, Ambrosio MR, Francini G, Calomino N, et al: Pathological response and outcome after neoadjuvant chemotherapy with DOC (docetaxel, oxaliplatin, capecitabine) or EOF (epirubicin, oxaliplatin, 5-fluorouracil) for clinical T3-T4 non-metastatic gastric cancer. Surg Oncol 32: 2-7, 2020.

43. Kurokawa Y, Doki Y, Mizusawa J, Terashima M, Katai H, Yoshikawa T, Kimura Y,TakiguchiS, Nishida Y,FukushimaN, et al: Bursectomy versus omentectomy alone for resectable gastric cancer (JCOG1001): A phase 3, open-label, randomised controlled trial. Lancet Gastroenterol Hepatol 3: 460-468, 2018

44. Dudekula DB, Panda AC, Grammatikakis I, De S, Abdelmohsen K and Gorospe M: CircInteractome: A web tool for exploring circular RNAs and their interacting proteins and microRNAs. RNA Biol 13: 34-42, 2016.

45. Zhong S, Wang J, Zhang Q, Xu H and Feng J: CircPrimer: A software for annotating circRNAs and determining the specificity of circRNA primers. BMC Bioinformatics 19: 292, 2018.

46. Vlachos IS, Zagganas K, Paraskevopoulou MD, Georgakilas G, Karagkouni D, Vergoulis T, Dalamagas T and Hatzigeorgiou AG: DIANA-miRPath v3.0: Deciphering microRNA function with experimental support. Nucleic Acids Res 43W: W460-W466, 2015.

47. Karagkouni D, Paraskevopoulou MD, Chatzopoulos S, Vlachos IS, Tastsoglou S, Kanellos I, Papadimitriou D, Kavakiotis I, Maniou S, Skoufos G, et al: DIANA-TarBase v8: A decade-long collection of experimentally supported miRNA-gene interactions. Nucleic Acids Res 46D: D239-D245, 2018.

48. Shannon P, Markiel A, Ozier O, Baliga NS, Wang JT, Ramage D, Amin N, Schwikowski B and Ideker T: Cytoscape: A software environment for integrated models of biomolecular interaction networks. Genome Res 13: 2498-2504, 2003.

49. Jia A, Xu L and Wang Y: Venn diagrams in bioinformatics. Brief Bioinform: Apr 12, 2021 (Epub ahead of print). doi: $10.1093 / \mathrm{bib} /$ bbab108

50. Thanh Huong P, Gurshaney S, Thanh Binh N, Gia Pham A, Hoang Nguyen $H$, Thanh Nguyen X, Pham-The H, Tran PT, Truong Vu K, Xuan Duong N, et al: Emerging Role of Circulating Tumor Cells in Gastric Cancer. Cancers (Basel) 12: 695, 2020.

51. Ning S, Wei W, Li J, Hou B, Zhong J, Xie Y, Liu H, Mo X, Chen J and Zhang L: Clinical significance and diagnostic capacity of serum TK1, CEA, CA 19-9 and CA 72-4 levels in gastric and colorectal cancer patients. J Cancer 9: 494-501, 2018. 
52. Wu D, Zhang P, Ma J, Xu J, Yang L, Xu W, Que H, Chen M and Xu H: Serum biomarker panels for the diagnosis of gastric cancer. Cancer Med 8: 1576-1583, 2019.

53. Shao Y, Tao X, Lu R, Zhang H, Ge J, Xiao B, Ye G and Guo J: hsa_circ_0065149 is an Indicator for Early Gastric Cancer Screening and Prognosis Prediction. Pathol Oncol Res 26: 1475-1482, 2020

54. Ding L, Zhao Y, Dang S, Wang Y, Li X, Yu X, Li Z, Wei J, Liu M and Li G: Circular RNA circ-DONSON facilitates gastric cancer growth and invasion via NURF complex dependent activation of transcription factor SOX4. Mol Cancer 18: 45, 2019.

55. Du W, Li D, Guo X, Li P, Li X, Tong S, Tong J, Kuang L and Liang D: Circ-PRMT5 promotes gastric cancer progression by sponging miR-145 and miR-1304 to upregulate MYC. Artif Cells Nanomed Biotechnol 47: 4120-4130, 2019.

56. Zhang YG, Yang HL, Long Y and Li WL: Circular RNA in blood corpuscles combined with plasma protein factor for early prediction of pre-eclampsia. BJOG 123: 2113-2118, 2016

57. Bahn JH, Zhang Q, Li F, Chan TM, Lin X, Kim Y, Wong DT and Xiao X: The landscape of microRNA, Piwi-interacting RNA, and circular RNA in human saliva. Clin Chem 61: 221-230, 2015

58. Wang Y, Mo Y, Gong Z, Yang X, Yang M, Zhang S, Xiong F, Xiang B, Zhou M, Liao Q, et al: Circular RNAs in human cancer. Mol Cancer 16: 25, 2017.

59. Zhao Z, Li X, Gao C, Jian D, Hao P, Rao L and Li M: Peripheral blood circular RNA hsa_circ_0124644 can be used as a diagnostic biomarker of coronary artery disease. Sci Rep 7: 39918, 2017.

60. Lin J, Cai D, Li W, Yu T, Mao H, Jiang S and Xiao B: Plasma circular RNA panel acts as a novel diagnostic biomarker for colorectal cancer. Clin Biochem 74: 60-68, 2019.

61. van Hootegem SJM, Smithers BM, Gotley DC, Brosda S, Thomson IG, Thomas JM, Gartside M, van Lanschot JJB, Lagarde SM, Wijnhoven BPL, et al: The Impact of Signet Ring Cell Differentiation on Outcome in Patients with Esophageal and Gastroesophageal Junction Adenocarcinoma. Ann Surg Oncol 26: 2375-2384, 2019.

62. Gunjigake K, Kinoshita J, Yamaguchi T, Saito H, Fujimori D, Horiike T, Harada S, Tajima H, Ninomiya I, Ohta T and Fushida S: Interleukin-17A derived from mast cells contributes to fibrosis in gastric cancer with peritoneal dissemination. Gastric Cancer 24: 31-44, 2021.
63. Chen Y, Zhou Q, Wang H, Zhuo W, Ding Y, Lu J, Wu G, Xu N and Teng L: Predicting Peritoneal Dissemination of Gastric Cancer in the Era of Precision Medicine: Molecular Characterization and Biomarkers. Cancers (Basel) 12: 2236, 2020.

64. Zhang S, Zang D, Cheng Y, Li Z, Yang B, Guo T, Liu Y, Qu X and Che X: Identification of Key Gene and Pathways for the Prediction of Peritoneal Metastasis of Gastric Cancer by Co-expression Analysis. J Cancer 11: 3041-3051, 2020.

65. Cheng L, Chen S, Wu W, Kuo ZC, Wei Z, Meng S, Chen C, Zhang $\mathrm{C}$ and $\mathrm{He} \mathrm{Y}$ : Gastric cancer in young patients: A separate entity with aggressive features and poor prognosis. J Cancer Res Clin Oncol 146: 2937-2947, 2020.

66. Endo S, Nishikawa K, Ikenaga M, Fujitani K, Kawada J, Yamatsuji T, Kubota H, Higashida M, Fujiwara Y and Ueno T: Prognostic factors for cytology-positive gastric cancer: A multicenter retrospective analysis. Int J Clin Oncol 26: 858-866, 2021.

67. Endo S, Ikenaga M, Ohta K, Ueda M, Tsuda Y, Kato R, Itakura H, Matsuyama J, Nishikawa K and Yamada T: Prognostic factors for cytology-positive gastric cancer. Surg Today 49: 56-64, 2019.

68. Shim HJ, Kim HJ, Lee SH, Bae WK, Hwang EC, Cho SH, Chung IJ, Bang HJ and Hwang JE: Observational Study of Peritoneal Washing Cytology-Positive Gastric Cancer without Gross Peritoneal Metastasis in Patients who Underwent Radical D2 Gastrectomy. Sci Rep 10: 9549, 2020.

69. Li X, Yang L and Chen LL: The Biogenesis, Functions, and Challenges of Circular RNAs. Mol Cell 71: 428-442, 2018.

70. Chen LL and Yang L: Regulation of circRNA biogenesis. RNA Biol 12: 381-388, 2015.

71. Bartel DP: MicroRNAs: Target recognition and regulatory functions. Cell 136: 215-233, 2009.

72. Li D, Zhang J and Li J: Role of miRNA sponges in hepatocellular carcinoma. Clin Chim Acta 500: 10-19, 2020.

73. Yang G, Zhang Y and Yang J: Identification of Potentially Functional circRNA-miRNA-mRNA Regulatory Network in Gastric Carcinoma using Bioinformatics Analysis. Med Sci Monit 25: 8777-8796, 2019.

This work is licensed under a Creative Commons Attribution-NonCommercial-NoDerivatives 4.0 International (CC BY-NC-ND 4.0) License. 